\title{
Prolidase deficiency, a rare inborn error of immunity, clinical phenotypes, immunological features, and proposed treatments in twins
}

Nora Alrumayyan ${ }^{1}$, Drew Slauenwhite', Sarah M. McAlpine1, Sarah Roberts', Thomas B. Issekutz', Adam M. Huber ${ }^{2}$, Zaiping Liu $^{3}$ and Beata Derfalvi ${ }^{*}$ (i)

\begin{abstract}
Background: Prolidase deficiency (PD) is an autosomal recessive inborn multisystemic disease caused by mutations in the PEPD gene encoding the enzyme prolidase $\mathrm{D}$, leading to defects in turnover of proline-containing proteins, such as collagen. PD is categorized as a metabolic disease, but also as an inborn error of immunity. PD presents with a range of findings including dysmorphic features, intellectual disabilities, recurrent infections, intractable skin ulceration, autoimmunity, and splenomegaly. Despite symptoms of immune dysregulation, only very limited immunologic assessments have been reported and standard therapies for PD have not been described. We report twin females with PD, including comprehensive immunologic profiles and treatment modalities used.
\end{abstract}

Case presentation: Patient 1 had recurrent infections in childhood. At age 13, she presented with telangiectasia, followed by painful, refractory skin ulcerations on her lower limbs, where skin biopsy excluded vasculitis. She had typical dysmorphic features of PD. Next-generation sequencing revealed pathogenic compound heterozygous mutations (premature stop codons) in the PEPD gene. Patient 2 had the same mutations, typical PD facial features, atopy, and telangiectasias, but no skin ulceration. Both patients had imidodipeptiduria. Lymphocyte subset analysis revealed low-normal frequency of $\mathrm{T}_{\text {reg }}$ cells and decreased frequency of expression of the checkpoint molecule CTLA-4 in CD4 ${ }^{+} \mathrm{T}_{\mathrm{EM}}$ cells. Analysis of Th1, Th2, and Th17 profiles revealed increased inflammatory IL-17 ${ }^{+} \mathrm{CD} 8^{+} \mathrm{T}_{\mathrm{EM}}$ cells in both patients and overexpression of the activation marker HLA-DR on CD4 ${ }^{+} \mathrm{T}_{E M}$ cells, reflecting a highly activated proinflammatory state. Neither PD patient had specific antibody deficiencies despite low $C D 4^{+} \mathrm{CXCR}^{+} \mathrm{T}_{\text {fh }}$ cells and low class-switched memory B cells. Plasma IL-18 levels were exceptionally high.

Conclusions: Immunologic abnormalities including skewed frequencies of activated inflammatory $\mathrm{CD} 4^{+}$and $\mathrm{CD} 8^{+}$ $T_{E M}$ cells, decreased CTLA-4 expression, and defects in memory B cells may be a feature of immune dysregulation associated with PD; however, a larger sample size is required to validate these findings. The high IL-18 plasma levels suggest underlying autoinflammatory processes.

Keywords: Prolidase deficiency, T cells, Inborn error of immunity, Autoimmunity, Leg ulcers

*Correspondence: beata.derfalvi@iwk.nshealth.ca

1 Division of Immunology, Department of Paediatrics, Dalhousie University, IWK Health Centre, Halifax, Canada

Full list of author information is available at the end of the article

\section{Background}

Prolidase deficiency (PD) (OMIM170100) is an autosomal recessive inborn error of metabolism caused by mutations in the PEPD gene encoding the enzyme original author(s) and the source, provide a link to the Creative Commons licence, and indicate if changes were made. The images or other third party material in this article are included in the article's Creative Commons licence, unless indicated otherwise in a credit line to the material. If material is not included in the article's Creative Commons licence and your intended use is not permitted by statutory regulation or exceeds the permitted use, you will need to obtain permission directly from the copyright holder. To view a copy of this licence, visit http://creativecommons.org/licenses/by/4.0/. The Creative Commons Public Domain Dedication waiver (http://creativeco mmons.org/publicdomain/zero/1.0/) applies to the data made available in this article, unless otherwise stated in a credit line to the data. 
prolidase $\mathrm{D}$, leading to defects in turnover of collagen and other proline-containing proteins. Since it was first described in 1968 by Goodman [1], 35 causative mutant alleles have been reported in the ninety cases worldwide [2, 3]. The incidence of $\mathrm{PD}$ is estimated to be 1-2 per million births, but it is more frequent in some populations, including the Druze and Arab Muslim minorities in Israel [2-6]. PD is categorized under Diseases of Immune Dysregulation, Syndromes with Autoimmunity in the 2019 IUIS Phenotypical Classification of Human Inborn Errors of Immunity [7]. PD is often referred to as a "lupus mimic", presenting predominantly with cutaneous features and with systemic autoimmunity [8-12]. Typical presentation of PD includes intractable skin ulceration, telangiectasia, recurrent infections, splenomegaly, dysmorphic features, and intellectual disabilities [2, 13]. Thrombocytopenia, hypocomplementemia, and hypergammaglobulinemia are frequent laboratory findings, as are increased levels of proline metabolites in serum and urine [9, 14-18].

To date, no standard diagnostic criteria or testing algorithms for PD have been published. PD is diagnosed by the detection of either biallelic PEPD pathogenic variants or reduced prolidase enzyme activity in patients who present with characteristic clinical findings and imidodipeptiduria $[2,17,19]$. The aim of this study was to identify features associated with immune dysregulation in PD patients through comprehensive immunologic assessments.

\section{Case presentation}

The index case, Patient 1 (Pt1), had recurrent ear and throat infections in early childhood, which when treated with antibiotics, tonsillectomy, adenoidectomy, and bilateral myringotomy tube insertion, lead to less frequent infections. At 13 years of age, Pt1 presented with severe, chronic, refractory skin ulcerations on her lower extremities, which prevented ambulation. Patient 2 (Pt2), an identical twin of Pt1, presented with recurrent respiratory tract infections as well as severe atopy, including anaphylaxis to multiple food allergens, allergic rhinitis, and asthma. Pt2 also had recurrent arthralgia, mainly in the large joints, with no stiffness or limitation of movement. No skin ulceration was present in Pt2. Both Pt1 and Pt2 presented with telangiectasia predominantly in their lower extremities. The clinical findings of both patients are summarized in Table 1.

Physical examination at 15 years of age showed that both twins had dysmorphic features, including a low hairline, mild ptosis, hypertelorism, a depressed nasal root, a beak-like nose, and micrognathia (Fig. 1). Pt1 had ulcers on the feet at different stages of healing with white discoloration ("atrophie blanche") of the skin around the ulceration (Fig. 2). Pt1 also had splenomegaly. Musculoskeletal examination was normal in both cases, except for observed hypermobility in Pt2. A sole skin punch biopsy was performed for Pt1 and excluded vasculitis but revealed livedoid vasculopathy with perivascular lymphocytic infiltration (Fig. 3). Poor wound healing was observed as the biopsy site did not heal for one year. Pt1 was diagnosed with PD at the age of 15 following next generation sequencing of 298 primary immunodeficiency genes (Blueprint Genetics). Previously reported pathogenic compound heterozygous mutations (c.977G $>$ A, p.Trp326* and c.550C $>$ T; p.Arg184*), which introduced premature stop codons in the PEPD gene, were detected $[20,21]$. Pt2 was found to have the same mutations. The non-consanguineous asymptomatic parents are carriers of one of the PEPD mutant variants, confirming trans position of the

Table 1 Clinical presentation in twin females with PD

\begin{tabular}{lll}
\hline Known clinical presentation of PD & Patient $\mathbf{1}$ & Patient $\mathbf{2}$ \\
\hline Skin manifestations & Diffuse telangiectasias & Telangiectasias one foot ulcer after 2 years \\
& Ulceration of the feet & + \\
Dysmorphic features & + & + \\
Intellectual disability (ID) & + & + \\
Recurrent infections & + & - \\
Coexistence autoimmunity & Euthyroid autoimmune thyroiditis & ++ Bronchial asthma, allergic rhinitis, \\
Allergic symptoms and atopy & - & eczema, food allergy \\
& - & -
\end{tabular}

+, clinical feature present; -, clinical feature absent 


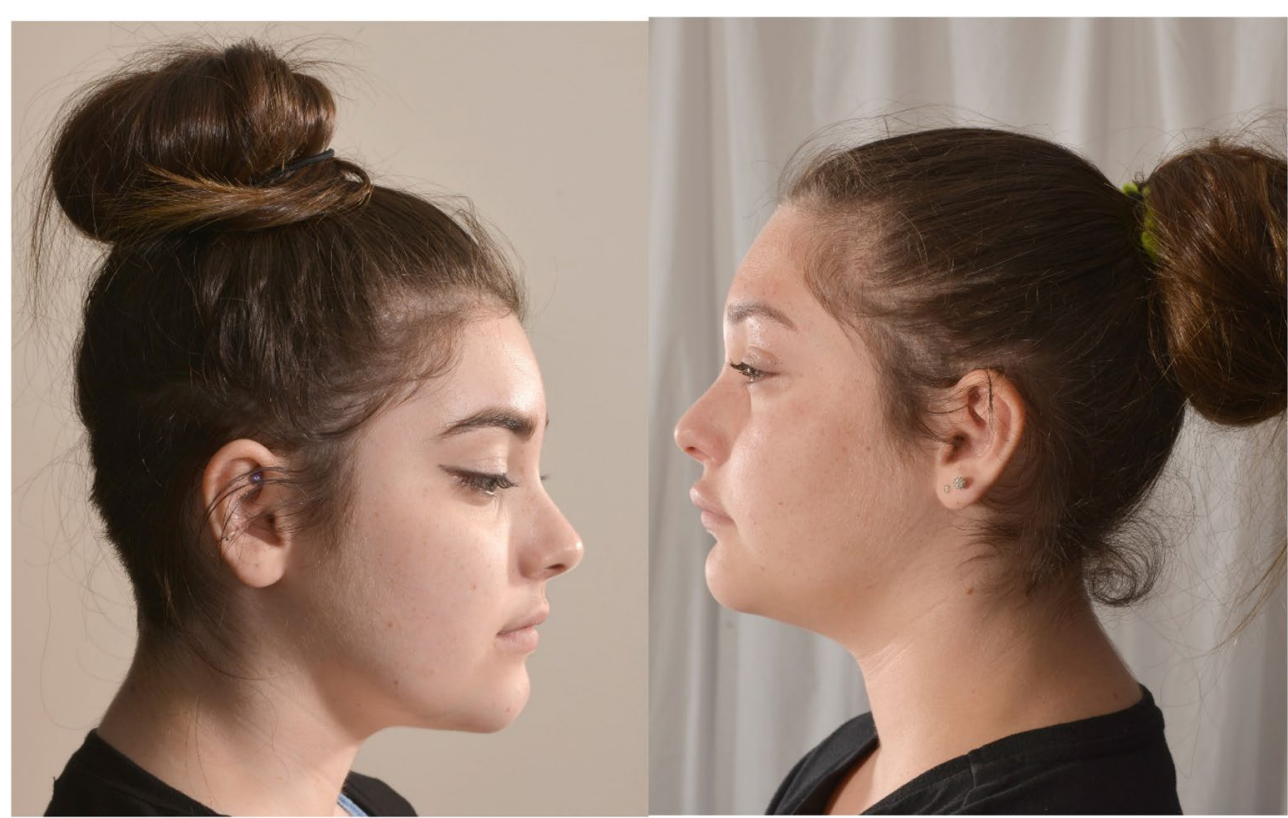

Panel A

Panel B

Fig. 1 Dysmorphic facial features in Patient 1 (Panel A) and patient 2 (Panel B) include low hairline, mild ptosis, hypertelorism, depressed nasal root, beak-like nose, and micrognathia

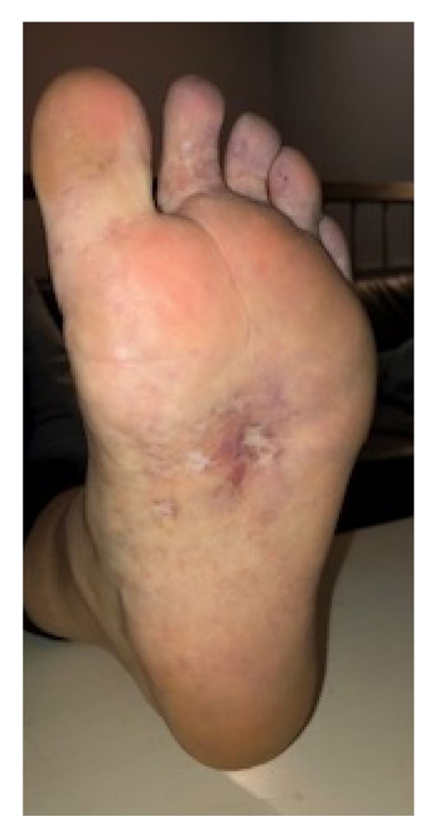

Fig. 2 Multiple ulcers at different stages of healing on the foot of Patient 1

mutated alleles. Imidodipeptiduria, indicating increased proline metabolites, confirmed PD in both patients [22] (Fig. 4). Family history for immune-mediated diseases was unremarkable except for maternal psoriasis and recurrent streptococcal infections.

Pt1 required gabapentin for pain control and antidepressants to treat severe anxiety, self-harm, and depression. She was started on low-molecularweight heparin (LMWH), pentoxifylline to improve microcirculation, and high dose $(1 \mathrm{~g} / \mathrm{kg})$ intravenous immunoglobulin (IVIG) infusions for immune modulation. This resulted in the cessation of disease progression and partial healing of ulcers. After genetic diagnosis, more targeted treatments were added, including high-dose collagen synthesis cofactor vitamin C $(500 \mathrm{mg} /$ day), 5\% proline-glycine ointment, and topical tacrolimus (0.33\%). Pt1 responded well to this combined therapy initially, allowing for recovery of full physical activity, and showed no new ulceration for 4 months. All previous ulcerations closed, leaving scars and whitish discoloration. Pt1 subsequently developed one new foot ulcer, which did not impact mobility and began to heal more quickly than previous ulcers.

Pt2 was started on high dose vitamin $C$ and pentoxifylline after presenting with white skin atrophy on the feet and mutation within PEPD was confirmed. One year later, Pt2 developed a small ulceration which was treated immediately with topical tacrolimus $(0.33 \%)$ and $5 \%$ proline-glycine ointment, which stopped ulcer progression and promoted healing. 


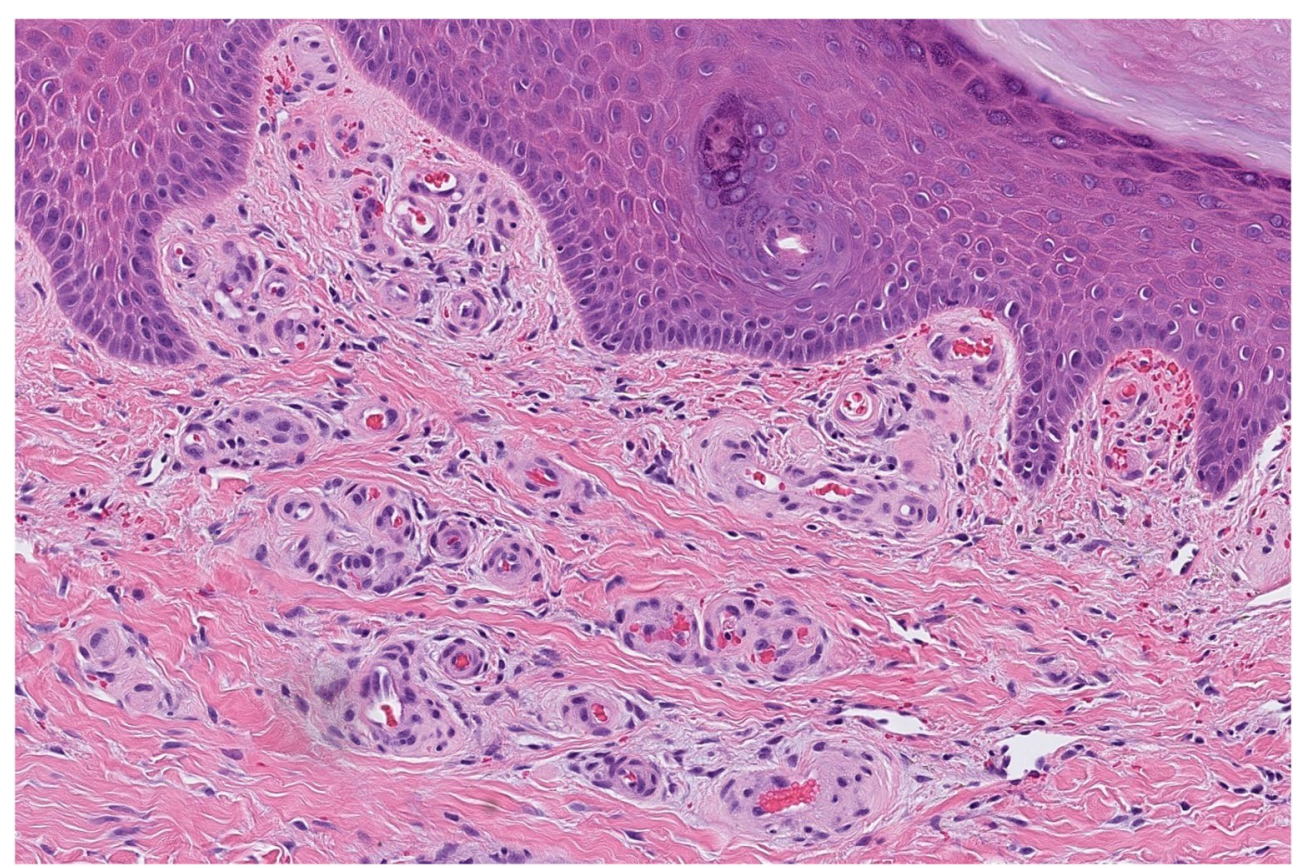

Fig. 3 Skin punch biopsy (sole) in Patient 1. Lobulated proliferation of capillaries, hemorrhage in dermis. The overlying epidermis is normal. No vasculitis. Hematoxylin-Eosin staining, $\times 400$

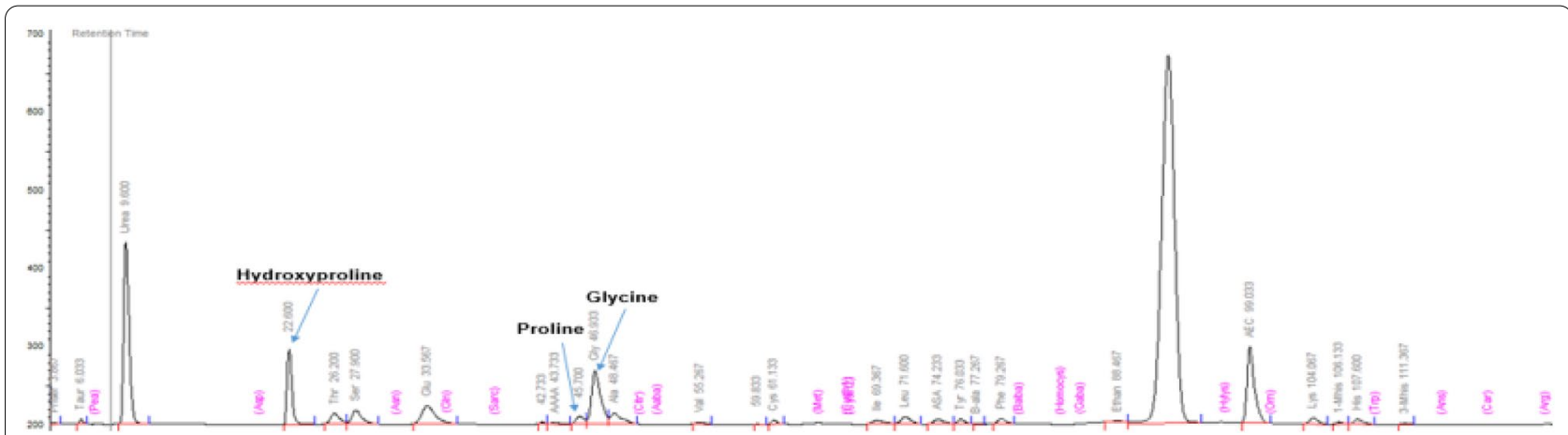

Fig. 4 Imidodipeptiduria, shown by increased Proline, Hydroxyproline and Glycine on urine amino acid quantitation by lon-Exchange Chromatography (Biochrom amino acid analyzer)

Magnetic resonance imaging (MRI) of the brain was performed for Pt1 due to chronic headaches, to rule out vasculitis, with normal results. Abdominal ultrasound showed splenomegaly in Pt1, which has been previously reported in PD [2, 3, 23], as well as hepatomegaly and diffuse hepatic steatosis in Pt1 and Pt2. The serum lipid profile revealed elevated low-density lipoprotein (LDL) and triglyceride levels in both patients, which has not been reported previously in PD. Pt1 had an overall low Wechsler Intelligence Scale for children-5th edition (IPad) (WISC-V) IQ score at the 5th percentile when tested at age 16, and she performed at the level of a
12 -year-old on the vocabulary test. The WISC-V IQ score of Pt2 ranged from extremely low to low average in the various test components.

A detailed laboratory assessment was conducted including measurement of humoral immunity, three pathways of complement activation, $\mathrm{T}$ and $\mathrm{B}$ lymphocyte proliferation to mitogens and antigens, and natural killer (NK) cell cytotoxicity. Findings of laboratory investigations are detailed in Tables 2 and 3. A complete blood count showed mild thrombocytopenia in Pt1. Immunoglobulin (total IgG, IgG1-4, IgA, IgM, IgE) levels were normal with the exception of low IgG2 in Pt2. Total 
Table 2 Clinical laboratory results in twin females with PD

\begin{tabular}{|c|c|c|c|}
\hline Test & Patient 1 & Patient 2 & Normal range \\
\hline \multicolumn{4}{|l|}{ Blood cell counts } \\
\hline White blood cells & 7.26 & 8.13 & 4.19-9.43 10e9/L \\
\hline Neutrophils & 2.2 & 4.22 & 1.82-7.47 10e9/L \\
\hline Lymphocytes & $4.09(H)$ & 2.9 & $1.16-3.33$ 10e9/L \\
\hline Monocytes & $0.73(H)$ & $0.76(H)$ & $0.19-0.72$ 10e9/L \\
\hline Platelets $^{a}$ & $118(L)$ & 154 & 130-400 10e9/L \\
\hline Mean platelet volume & 9.8 & 10.3 & $8-12 f L$ \\
\hline Hemoglobin & 130 & 149 & $105-150 \mathrm{~g} / \mathrm{L}$ \\
\hline \multicolumn{4}{|l|}{ Immunoglobulins } \\
\hline $\lg A$ & 0.81 & 1.35 & $0.52-1.92 \mathrm{~g} / \mathrm{L}$ \\
\hline $\lg M$ & 0.63 & 1.07 & $0.47-3.11 \mathrm{~g} / \mathrm{L}$ \\
\hline $\lg \mathrm{E}^{\mathrm{b}}$ & 423 & 568 & $<629 \mathrm{kU} / \mathrm{L}$ \\
\hline $\lg G^{c}$ & 8.92 & 9.56 & $7-15.9 \mathrm{~g} / \mathrm{L}$ \\
\hline $\lg G 1$ & 5.77 & 7.54 & $3.15-8.55 \mathrm{~g} / \mathrm{L}$ \\
\hline $\lg G 2$ & $0.30(\mathrm{~L})$ & 0.65 & $0.64-4.95 \mathrm{~g} / \mathrm{L}$ \\
\hline $\lg G 3$ & 0.3 & 0.29 & $0.23-1.96 \mathrm{~g} / \mathrm{L}$ \\
\hline $\lg G 4$ & 0.158 & 0.384 & $0.11-1.57 \mathrm{~g} / \mathrm{L}$ \\
\hline $\begin{array}{l}\text { Specific antibody titers to diphtheria, } \\
\text { tetanus, pneumococcal, rubella, } \\
\text { varicella, and measles vaccine antigens }\end{array}$ & $\begin{array}{l}\text { Good, sustained, protective antibody } \\
\text { titers to vaccines except borderline } \\
\text { measles lgG response }\end{array}$ & $\begin{array}{l}\text { Good, sustained, protective } \\
\text { antibody titers to all vaccines }\end{array}$ & \\
\hline $\begin{array}{l}\text { B cell proliferation (\% of CpG-stimulated } \\
\text { cells divided) }\end{array}$ & $68.1 \%$ & $65.0 \%$ & $63.2-100 \%{ }^{d}$ \\
\hline $\begin{array}{l}\text { T cell proliferation: mitogen and antigen } \\
\text { stimulation of PBMCs by PHA, ConA, } \\
\text { PWM, anti-CD3, anti-CD3 + IL-2, IL-2, } \\
\text { tetanus toxoid, diphtheria toxoid, and } \\
\text { Candida albicans antigens }\end{array}$ & $\begin{array}{l}\text { Normal lymphocyte proliferation to all } \\
\text { mitogens and antigens tested }\end{array}$ & $\begin{array}{l}\text { Normal lymphocyte } \\
\text { proliferation to all mitogens } \\
\text { and antigens tested }\end{array}$ & \\
\hline \multicolumn{4}{|l|}{ NK cell function } \\
\hline $\begin{array}{l}\text { NK cell cytotoxicity (NK cell killing } \\
\text { activity) }\end{array}$ & Normal & Normal & \\
\hline Degranulation $\left(C D 107 a^{+}\right)$ & $27 \%$ & $22 \%$ & $11-35 \%$ \\
\hline Neutrophils NBT reduction & Normal oxidative burst of $99 \%$ & Normal oxidative burst of $100 \%$ & \\
\hline \multicolumn{4}{|l|}{ Complement } \\
\hline CH50 classical & 93 & 70 & $42-96 \mathrm{U} / \mathrm{mL}$ \\
\hline$C 3^{\mathrm{e}}$ & 1.54 & 1.63 & $1.1-1.8 \mathrm{~g} / \mathrm{L}$ \\
\hline$C 4^{e}$ & 0.26 & 0.24 & $0.17-0.39 \mathrm{~g} / \mathrm{L}$ \\
\hline MBL & $0(L)$ & $0(L)$ & $30-200 \%$ \\
\hline Alternate complement & 119 & 107 & $>40 \%$ \\
\hline \multicolumn{4}{|l|}{ Inflammatory markers } \\
\hline ESR & $18(H)$ & 2 & $0-9 \mathrm{~mm} / \mathrm{h}$ \\
\hline CRP & $24.6(H)$ & $6(\mathrm{H})$ & $0-5.0 \mathrm{mg} / \mathrm{L}$ \\
\hline Ferritin & $400(\mathrm{H})$ & $2006(H)$ & $5.5-67 \mathrm{mcg} / \mathrm{L}$ \\
\hline IL-18 & $>36,600(\mathrm{H})$ & $28,803(H)$ & $<266 \mathrm{pg} / \mathrm{mL}$ \\
\hline SAA & 9837 & 8511 & $\mathrm{ng} / \mathrm{mL}$, within $30-70 \%$ of normal \\
\hline \multicolumn{4}{|l|}{ Auto-antibodies $^{f}$} \\
\hline ANA & ANA IIF + (1:320, speckled pattern) & All negative & \\
\hline $\begin{array}{l}\text { ENA, anti-TTG-IgA, anti-cardiolipin, } \\
\text { anti-B2GP1, LA, ASMA, APCP, anti-LKM, } \\
\text { anti-PR3, anti-MPO, anti-CCP, RF, anti-TPO }\end{array}$ & anti-TPO+ + & & \\
\hline \multicolumn{4}{|l|}{ Lipid profile } \\
\hline LDL & $\mathrm{ND}^{\mathrm{g}}$ & 3.08 & $\begin{array}{l}\text { Acceptable }<2.85 \\
\mathrm{H}>3.36 \mathrm{mmol} / \mathrm{L}\end{array}$ \\
\hline
\end{tabular}


Table 2 (continued)

\begin{tabular}{llll}
\hline Test & Patient 1 & Patient 2 & Normal range \\
\hline HDL & $0.79(\mathrm{~L})$ & 1.2 & Acceptable $>1.17 \mathrm{mmol} / \mathrm{L}$ \\
TGA & $6.1(\mathrm{H})$ & $2.21(\mathrm{H})$ & Acceptable $<1.02$ \\
Cholesterol & $6.86(\mathrm{H})$ & $5.28(\mathrm{H})$ & $\mathrm{H}>1.46 \mathrm{mmol} / \mathrm{L}$ \\
& & & Acceptable $<4.40$ \\
\end{tabular}

$\mathrm{L}$, low; $\mathrm{H}$, high; PHA, phytohemagglutinin; ConA, concanavalin A; PWM, pokeweed mitogen; NK, natural killer cells; $M B L$, mannose-binding lectin; ESR, erythrocyte sedimentation rate; CRP, C-reactive protein; SAA, serum amyloid A; pc, percentile; ANA, antinuclear antibodies; ENA, extractable nuclear antigen antibodies; SS-A/B, Sjögren's-syndrome-related antigen A/B autoantibodies; anti-TTG-lgA, anti-tissue transglutaminase lgA antibodies; anti-B2GP1, anti-beta-2-glycoproteins antibodies; ASMA, anti-smooth muscle antibodies; ACPA, anti-citrullinated protein antibodies; anti-LKM, anti-liver-kidney-muscle antibodies; anti-MPO, anti-myeloperoxidase antibodies; anti-CCP, anti-cyclic citrullinated peptide antibodies; RF, rheumatoid factor; anti-TPO, anti-thyroid peroxidase antibodies; LDL, low-density lipoprotein; $\mathrm{HDL}$, high-density lipoprotein; TGA, triglycerides

a Thrombocytopenia reported in literature [1]

${ }^{\mathrm{b}}$ Increased serum IgE levels reported in literature [3]

c Hypergammaglobulinemia reported in literature [2]

${ }^{\mathrm{d}}$ Mean $\pm 2 \mathrm{SD}, \mathrm{n}=6$ healthy donors

e Hypocomplementemia (C3 and C4) reported in literature [3]

f Positive ANA, anti-dsDNA, anti-ENA (anti-Ro), anti-Sm, and anti-chromatin have been found in individuals with prolidase deficiency even in the absence of clinical findings of SLE [2]

${ }^{g}$ Not determined due to high TGA

B cell counts were normal and antibody titers to tested vaccine antigens were sustained as protective, confirming normal specific antibody production, despite both patients having low peripheral class-switched memory B cells. Lymphocyte proliferation in response to stimulation with mitogens and antigens was normal as detected via $\mathrm{H}^{3}$-thymidine incorporation assay. NK cell cytotoxicity, indicated by degranulation and target cell killing, was also normal. Both patients were mannose-binding lectin (MBL) deficient. Autoantibody measurements confirmed antinuclear antibody (ANA) and anti-thyroid peroxidase (TPO) antibody positivity in Pt1. Blood inflammatory parameters $\mathrm{C}$-reactive protein (CRP) and erythrocyte sedimentation rate (ESR) were elevated in Pt1, even when the patient's ulcers resolved.

Detailed immunophenotyping of $\mathrm{T}, \mathrm{B}$, and NK lymphocyte subsets and B cell function were further characterized by flow cytometry (BD LSR Fortessa SORP flow cytometer). Heparinized blood was collected from $\mathrm{Pt} 1, \mathrm{Pt} 2$ at 16 years of age, and healthy, adult volunteers $(\mathrm{n}=14$, mean age \pm SD $45.8 \pm 13.2$ years, 8 females and 6 males), with no history of infections or autoimmune disease. Peripheral blood mononuclear cells (PBMCs) were isolated using Ficoll-Paque PLUS (GE Healthcare) and cryopreserved in liquid nitrogen. To evaluate $\mathrm{B}$ cell function, patient PBMCs were labelled with CellTrace and stimulated with the Toll-like receptor 9 agonist CpG-oligodeoxynucleotide. After 6 days, flow cytometric analysis revealed $B$ cell proliferation in both patients were within the range observed in healthy donors.

NK and T lymphocyte immunophenotyping revealed normal NK and $\mathrm{T}$ cell subsets, including a normal $\mathrm{T}$ cell $\mathrm{CD}^{+} / \mathrm{CD}^{+}$ratio and no increase in doublenegative $\mathrm{T}$ cells $\left(\mathrm{CD} 3^{+} \mathrm{CD} 4^{-} \mathrm{CD} 8^{-} \mathrm{TCR} \alpha \beta^{+}\right) . \mathrm{CD} 4^{+}$ and $\mathrm{CD}^{+} \mathrm{T}$ cell subsets were identified as previously described [24], based on CCR7 and CD45RA expression: naïve, effector $\left(\mathrm{T}_{\text {eff }}\right)$, central memory $\left(\mathrm{T}_{\mathrm{CM}}\right)$, effector memory $\left(\mathrm{T}_{\mathrm{EM}}\right)$, and effector memory re-expressing CD45RA ( $\left.\mathrm{T}_{\mathrm{EMRA}}\right)$. Both patients had lower frequencies of $\mathrm{CD} 4^{+} \mathrm{T}_{\mathrm{EMRA}}$ and $\mathrm{CXCR} 5^{+} \mathrm{CD} 4^{+}$ $\mathrm{T}$ follicular helper $\left(\mathrm{T}_{\mathrm{fh}}\right)$ cells compared to healthy controls. Pt1 had a higher proportion of $\mathrm{CD}^{+} \mathrm{T}_{\mathrm{CM}}$ cells among total $\mathrm{CD} 4^{+} \mathrm{T}$ cells and a higher frequency of $\mathrm{CD}^{+}$memory $\mathrm{T}$ cells, $\mathrm{T}_{\mathrm{CM}}$, and $\mathrm{T}_{\mathrm{EMRA}}$, among total $\mathrm{CD}^{+} \mathrm{T}$ cells (Table 3 ). To examine the profile of T helper type 1 (Th1), Th2, and Th17 cells, patient PBMCs were stimulated with PMA and ionomycin for $3 \mathrm{~h}$ and intracellular cytokine staining was performed for interferon- $\gamma$ (IFN- $\gamma$ ), interleukin-13 (IL-13), and IL-17, respectively. Both patients had increased proportions of $\mathrm{IL}-17^{+} \mathrm{CD} 8^{+} \mathrm{T}_{\mathrm{EM}}$ cells, compared to healthy controls. Pt2 also had a lower proportion of IFN- $\gamma^{+}$cells among both $\mathrm{CD}^{+}$and $\mathrm{CD} 8^{+} \mathrm{T}_{\mathrm{EM}}$ cells. Compared to healthy controls, measuring expression of the checkpoint molecules TIM-3, CTLA-4, and PD-1 revealed higher TIM- 3 expression among CD4 ${ }^{+}$ $\mathrm{T}_{\mathrm{EM}}$ cells and lower CTLA-4 expression in CD $4^{+} \mathrm{T}_{\mathrm{EM}}$ cells in both Pt1 and Pt2. Analysis of the activation marker HLA-DR showed increased expression on $\mathrm{CD} 4^{+} \mathrm{T}_{\mathrm{EM}}$ cells in both patients.

The plasma inflammatory cytokine profile and serum amyloid A (SAA) levels were measured using ELISA and a multiplex immunoassay (Human Cytokine 65-Plex Clinical RUO Discover). SAA and proinflammatory 
Table 3 Absolute counts and frequencies of $\mathrm{CD}^{+}{ }^{+} \mathrm{T}$ cell, $\mathrm{CD} 8^{+} \mathrm{T}$ cell, and $\mathrm{CD} 4^{+} \mathrm{T}_{\text {reg }}$ cell subsets in the peripheral blood of healthy controls and PD patients

\begin{tabular}{|c|c|c|c|c|}
\hline Cell type & Subset & Patient 1 & Patient 2 & $\begin{array}{l}\text { Normal age-related } \\
\text { range (clinical } \\
\text { laboratory) }\end{array}$ \\
\hline \multirow[t]{9}{*}{ Lymphocytes } & Absolute $\mathrm{CD}^{+}$ & 2394 & 2830 & 850-3200 cells/ $\mu \mathrm{L}$ \\
\hline & Absolute $\mathrm{CD}^{+}{ }^{+} \mathrm{CD} 4^{+}$ & 1406 & 2065 & $400-2100$ cells $/ \mu \mathrm{L}$ \\
\hline & Absolute $\mathrm{CD}^{+}{ }^{+} \mathrm{CD} 8^{+}$ & 1178 & 766 & 300-1300 cells/ $\mu \mathrm{L}$ \\
\hline & $\mathrm{CD}^{+} / \mathrm{CD}^{+}{ }^{+}$ratio & $1.1(\mathrm{~L})$ & 2.5 & $1.5-2.5$ \\
\hline & B cells $\left(C D 19^{+}\right)$ & 122 & 266 & 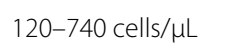 \\
\hline & $\%$ class-switched memory B cells & $2 \%(L)$ & $4 \%(L)$ & $9-26 \%$ \\
\hline & NK cells & $1102(H)$ & 133 & 7-480 cells/ $\mu \mathrm{L}$ \\
\hline & $\mathrm{CD}^{+}{ }^{+} \mathrm{CD} 4^{-} \mathrm{CD} 8^{-}$alpha/beta & $0.6 \%$ & $0.8 \%$ & $<1.5 \%$ \\
\hline & & & & Normal range ${ }^{*}$ \\
\hline \multirow[t]{6}{*}{$\mathrm{CD}^{+}{ }^{+} \mathrm{T}$ cells } & $\begin{array}{l}\mathrm{CD}^{+} \text {memory } \\
\left(\mathrm{CD} 45 \mathrm{RO}^{+}\right)\end{array}$ & 33.1 & $19.1(L)$ & $23.4-66.5$ \\
\hline & $\mathrm{CD} 4^{+} \mathrm{T}_{\mathrm{EM}}$ & 21.5 & 11.4 & $9.1-48.4$ \\
\hline & $\mathrm{CD}^{+} \mathrm{T}_{\mathrm{CM}}$ & $9.0(H)$ & 5.3 & $2.1-7.5$ \\
\hline & $\mathrm{CD}^{+} \mathrm{T}_{\text {EMRA }}$ & $2.1(\mathrm{~L})$ & $1.8(\llcorner)$ & $3.0-13.6$ \\
\hline & $\mathrm{CD} 4^{+} \mathrm{T}_{\text {eff }}$ & 0.3 & 0.5 & $0-2.5$ \\
\hline & $\mathrm{CD}^{+}$naïve $\left(\mathrm{CD} 4 \mathrm{RA}{ }^{+}\right)$ & 59.1 & $74.2(H)$ & $21.2-62.7$ \\
\hline \multirow[t]{6}{*}{$\mathrm{CD}^{+} \mathrm{T}$ cells } & $\mathrm{CD}^{+}$memory $\left(\mathrm{CD} 45 \mathrm{RO}^{+}\right)$ & $77.3(\mathrm{H})$ & 39.4 & $8.2-68.3$ \\
\hline & $\mathrm{CD} 8^{+} \mathrm{T}_{\mathrm{EM}}$ & 30.0 & 14.7 & $0-30.7$ \\
\hline & $\mathrm{CD}^{+} \mathrm{T}_{\mathrm{CM}}$ & $2.6(H)$ & 0.7 & $0.1-2.5$ \\
\hline & $\mathrm{CD}^{+} \mathrm{T}_{\text {EMRA }}$ & $43.6(H)$ & 20.7 & $8.7-29.0$ \\
\hline & $\mathrm{CD} 8^{+} \mathrm{T}_{\text {eff }}$ & 2.4 & 2.5 & $0-29.4$ \\
\hline & $\mathrm{CD}^{+}$naïve $\left(\mathrm{CD}^{2} 5 \mathrm{RA}^{+}\right)$ & 15.2 & 54.6 & $8.3-83.9$ \\
\hline \multirow[t]{2}{*}{ T follicular cells } & $\mathrm{CD}^{+}{ }^{+} \mathrm{CXCR}^{+}$ & $1.8(\mathrm{~L})$ & $2.0(\llcorner)$ & $2.2-9.4$ \\
\hline & $\mathrm{CD}^{+} \mathrm{CXCR5}^{+}$ & 0.3 & 0.2 & $0-4.4$ \\
\hline \multirow[t]{3}{*}{$\mathrm{T}_{\text {reg }}$ cells } & Total $\mathrm{T}_{\text {reg }}\left(\%\right.$ of $\left.\mathrm{CD}^{+}\right)$ & 3.6 & 2.8 & $2.6-6.3$ \\
\hline & Naive $T_{\text {reg }}\left(C D 45 \mathrm{RO}^{-}\right)$ & 27.1 & 38.9 & $15.1-49.4$ \\
\hline & Memory reg $\left(C D 45 \mathrm{RO}^{+}\right)$ & 57.2 & 41.8 & $40-82$ \\
\hline \multirow[t]{6}{*}{ T cell cytokines } & $\mathrm{CD}^{+} \mathrm{T}_{\mathrm{EM}} \mathrm{IFN}-\gamma^{+}$ & 37.5 & $9.3(\llcorner)$ & $12.8-56.4$ \\
\hline & $\mathrm{CD}^{+} \mathrm{T}_{\mathrm{EM}} \mathrm{IL}-13^{+}$ & 3.0 & 6.8 & $2.3-7.0$ \\
\hline & $\mathrm{CD}^{+} \mathrm{T}_{\mathrm{EM}} \mathrm{IL}-17^{+}$ & 1.8 & 2.4 & $1.0-3.2$ \\
\hline & $\mathrm{CD}^{+} \mathrm{T}_{\mathrm{EM}} \mathrm{IFN}-\gamma^{+}$ & 55.2 & $35.5(L)$ & $42.0-100$ \\
\hline & $\mathrm{CD} 8^{+} \mathrm{T}_{\mathrm{EM}} \mathrm{IL}-13^{+}$ & 0.7 & 0.4 & $0.2-8.1$ \\
\hline & $\mathrm{CD}^{+} \mathrm{T}_{\mathrm{EM}} \mathrm{IL}-17^{+}$ & $1.7(\mathrm{H})$ & $1.4(\mathrm{H})$ & $0-0.9$ \\
\hline \multirow[t]{3}{*}{ Checkpoint molecules } & $\mathrm{CD}^{+}{ }^{+} \mathrm{T}_{\mathrm{EM}} \mathrm{CTLA}^{+} 4^{+}$ & $22.4(L)$ & $21.2(\mathrm{~L})$ & $24.5-43.7^{* *}$ \\
\hline & $\mathrm{CD}^{+}{ }^{+} \mathrm{T}_{\mathrm{EM}}{\mathrm{PD}-1^{+}}^{+}$ & 58.5 & 44.9 & $26.6-70.3$ \\
\hline & $\mathrm{CD}^{+} \mathrm{T}_{\mathrm{EM}} \mathrm{TIM}^{+} 3^{+}$ & $3.7(H)$ & $4.8(H)$ & $0.4-1.8$ \\
\hline \multirow[t]{2}{*}{ Activation marker } & $\mathrm{CD}^{+}{ }^{+} \mathrm{T}_{\mathrm{EM}} \mathrm{HLA}-\mathrm{DR}^{+}$ & $20.2(H)$ & $10.2(H)$ & $1.5-9.4$ \\
\hline & $\mathrm{CD}^{+} \mathrm{T}_{\mathrm{EM}} \mathrm{HLA}-\mathrm{DR}^{+}$ & 6.3 & 7.5 & $0-22.5$ \\
\hline
\end{tabular}

Information on the optimized antibody panels used for flow cytometry can be obtained by contacting the corresponding author. Numbers represent the percentage of cells comprising each subset or percentage of cells expressing the indicated markers.

$\mathrm{T}_{\mathrm{CM}}$, central memory $\mathrm{T}$ cells; $\mathrm{T}_{\text {eff }}$, effector $\mathrm{T}$ cells; $\mathrm{T}_{\mathrm{EM}}$, effector memory $\mathrm{T}$ cells; $\mathrm{T}_{\mathrm{EMRA}}$, $\mathrm{T}$ cell re-expressing $\mathrm{CD} 45 \mathrm{RA}$; $\mathrm{H}$, higher than normal range; $\mathrm{L}$, lower than normal range

*The normal range is represented by mean $\pm 2 \times$ standard deviation of $n=7$ healthy controls, mean age \pm SD $34.3 \pm 4.9$ years and ${ }^{* *}$ mean age \pm SD $54.8 \pm 10.8$ years. $\mathrm{T}_{\text {reg }}$ cells were defined as $\mathrm{CD} 3^{+} \mathrm{CD} 4^{+} \mathrm{CD} 127^{\text {low/- }} \mathrm{CD} 25^{\text {high }} \mathrm{FoxP} 3^{+}$. 
cytokines, including IL-1 $\beta$, IL-6, IL-17A, and tumor necrosis factor (TNF), were within the normal range (1597.5\% distribution) in both patients, except for plasma IL-18 levels, which were increased more than 100-fold compared to healthy controls.

\section{Discussion}

Individuals with PD experience severe morbidity and early death, usually due to infection. To date, 90 patients with PD have been reported in the literature [3], and nine have passed away with infection as a leading cause. PD patients experience bacteremia, skin infections, and cellulitis, with frequent infection by influenza, Pseudomonas aeruginosa, and fungi, the latter of which are infections typically observed in combined or innate immune deficiencies [14, 25]. Decreased neutrophil chemotaxis was reported [26], however we did not assess this in this study. Pt1 and Pt2 presented with recurrent respiratory tract infections with MBL complement deficiency being a possible contributing factor. Moreover, prolidase is required for the maturation of type I IFN receptors [27]. PD patients lack PEPD activity, leading to inhibition of type I IFN receptor-dependent immune responses, which are critical for the amplification of innate immunity and the mobilization of adaptive immunity in response to infection.

CXCR $5^{+} \mathrm{CD} 4^{+} \mathrm{T}_{\mathrm{fh}}$ cells are specialized cells that provide help to $\mathrm{B}$ cells especially during affinity maturation which is essential for high-affinity antibody production and the development of memory B cells [28] Laboratory measured immune abnormalities reported in the literature include elevated levels of IgG, IgA, IgM, and IgE, deficiency of the complement component C1q, and low C3 and C4 complement levels [13, 14, 26, 29]. Hypergammaglobulinaemia is likely secondary to recurrent infections or immune dysregulation [26, 29, 30]. The low proportion of $T_{\text {fh }}$ cells in both patients may contribute to low class-switched memory B cells. Despite these observations, specific antibody production was intact as well as B cell proliferation to $\mathrm{T}$ cell-independent stimulation. It is possible that low circulating $\mathrm{T}_{\mathrm{fh}}$ cells may not be reflective of lymph node-resident $T_{\text {fh }}$ cell populations as the former have been shown to be phenotypically distinct from their counterparts in the tissue [31]. Indeed, mechanisms of immune deficiency in the patients in the present study do not appear to involve germinal center B cell responses or the development of effective humoral immunity.

Reports of immune dysregulation in PD patients have been published, including a patient with very early onset inflammatory bowel disease [32]. Additionally, association between systemic lupus erythematosus (SLE) and PD has been described $[8-10,12,14,25,33]$ with common symptoms including anemia, thrombocytopenia, hypergammaglobulinemia, hypocomplementemia, and elevated titers of autoantibodies [2]. Pt1 had ANA positivity and anti-TPO autoantibodies. We focused on immune checkpoint molecules and cytokines expressed among effector memory $\mathrm{T}$ cells, since naïve $\mathrm{T}$ cells express very low levels in both health and disease. We have observed a deficiency of CD4 ${ }^{+} \mathrm{T}_{\mathrm{EM}}$ CTLA- $4^{+}$cells and borderline low $\mathrm{T}_{\text {reg }}$ cell frequencies. Although this was relatively modest, even partial CTLA-4 deficiency results in severe autoimmune disease such as in CTLA-4 haploinsufficiency with autoimmune infiltration (CHAI) [34]. In contrast, TIM-3 expression in CD4 ${ }^{+} \mathrm{T}_{\mathrm{EM}}$ cells was upregulated, which has been associated with reduced $\mathrm{T}$ cell inflammatory responses, and favorable longterm outcomes in multiple autoimmune diseases. $\mathrm{T}$ cell cytokine responses in both patients were normal with the exception of elevated proportions of $\mathrm{IL}-17^{+} \mathrm{CD} 8^{+} \mathrm{T}_{\mathrm{EM}}$ cells [35]. Pt2, who presented with severe atopy, had low proportions of IFN- $\gamma$-producing $\mathrm{T}$ cells, a relationship which has been previously shown in patients with atopic disease [36]. Immune dysregulation is further evidenced by the chronically elevated CRP in Pt1 and very high IL-18 levels in both patients suggesting an autoinflammatory process, like in the NLRC4 inflammasomerelated clinical disease spectrum [37]. Prolidase cleaves di- and tripeptides at the carboxyl terminal of proline resulting in a unique ring structure that prevents proteolysis and maintains the protein's biological activity $[38,39]$. Damaged proline in PD, as endogenous danger signal could lead to dysregulated inflammasome signaling and hyperinflammation. Another possible mechanism linking PD with dysregulated immune activity is through the nuclear factor kappa $\mathrm{B}(\mathrm{NFK} B)$ transcription factor, which represents a critical network for coordinating inflammatory responses. Prolidase activity is inversely associated with $\mathrm{NF} \mathrm{KB}$ activity. Intriguingly, the complexes which cooperate in the physiological activation or regulation of $\mathrm{NF} \mathrm{K} B$ activity vary in their roles depending upon the cell type or signaling pathway involved [40]. This may explain the diverse outcomes observed within and between individuals lacking prolidase. In some immune cells or tissue microenvironments, regulation of NFKB-induced inflammation by prolidase is lost while in others it remains intact through a network of tightly regulated compensatory mechanisms. Additional studies are needed to improve our understanding of the biochemistry of prolidase-dependent regulation of transcription factor activity before mechanisms for immune dysregulation in PD become clear.

Both patients had an abnormal lipid profile, which was unexpected as they had a normal BMI, were on a healthy diet, and had no family history of first degree 
coronary disease. Both had high cholesterol and LDL levels, hepatic steatosis, with hepatomegaly and elevated liver enzymes, which have not been previously observed in PD patients. Prolidase has been linked to lipid metabolism and may explain the hyperlipidemia findings, therefore indicating a need to monitor lipid levels in PD patients [41]. Intellectual disability has been reported in $71 \%$ of patients with PD [13]. The IQ scores reported vary from extremely low to low-average, in patients assessed by the WISC-V scale [3]. IQ scores of Pt1 and Pt2 were within the previously reported average for PD patients. The cause of intellectual disability in PD patients is not clear, but a recent study has proposed that PD may affect synaptic neurotransmission [42]. In addition, the basement membrane of the pial meninges (pBM), which is essential for brain cortical maturation, is predominantly composed of type IV collagen which would be negatively affected by PD due to disruptions in collagen metabolism [42].

Pt1 and Pt2 have a classical presentation of PD including dysmorphic facial features, intellectual disability, chronic, severe skin ulcers of the lower extremities, and telangiectasias. Pt1 presented with a more aggressive inflammatory disease with severe skin manifestations and autoimmunity, while Pt2 presented with more atopy. Different PD phenotypes between siblings have been previously reported and varying environmental factors or epigenetic modulators have been proposed as causative $[5,43,44]$.

The treatment of PD is symptomatic and there is no recommended or curative regimen. Topical and systemic treatments have been used with only partial improvement reported. Most treatment strategies aim to replace prolidase or treat and stop ulcerative progression. Topical treatments include proline, growth hormone (GH), and tacrolimus [45-48]. Topical proline ointment has been reported to rapidly reduce ulcer size, especially when combined with a $5 \%$ glycine ointment, but has not been shown to prevent new ulcers [2, 44, 47]. GH, LMWH, and collagen cofactor Vitamin C $(4 \mathrm{~g} / \mathrm{kg})$ have been previously shown to accelerate wound healing but has not been shown to prevent new ulcer development [38, 46, 49]. Vitamin C and LMWH have been shown to reduce symptoms where patients had evidence of thrombosis in the cutaneous microcirculation [2, 38, 44, 49-51], Topical tacrolimus, which is immunosuppressive and anti-inflammatory, was found to be very effective in treating ulcers without causing skin atrophy, however, did not prevent new ulcers [48]. Partially successful treatment with systemic immunosuppressive medications or frequent packed red blood cells transfusions have been also reported $[13,51]$. In a previous study of a PD patient, hematopoietic stem cell transplantation (HSCT) was performed with signs of successful engraftment, however, the patient developed a severe infection and unfortunately died [52]. Besides monitoring the physical changes to determine clinical response to variable treatments, more objective methods to determine successful treatment could include measuring proline urine metabolism or erythrocyte prolidase levels $[13,51]$.

The limitations of this study include the use of peripheral blood mononuclear cells without paired tissue biopsies from sites such as the skin or lymph nodes where the balance of inflammatory and immunoregulatory processes may be different. Moreover, immunophenotyping of patients occurred after Pt1 was offered IVIG as disease activity was high preceding the blood sample collection date, which may have influenced the results. Immunophenotyping data were compared to a limited number of healthy controls which were not matched for age or sex, therefore a larger study is required to replicate these findings.

\section{Conclusion}

Prolidase deficiency is a complex disorder with a broad clinical spectrum of presentations and severity. PD, with its immune components, may be classified as a primary immune regulatory disorder (PIRD). Very high IL-18 plasma levels suggest underlying autoinflammatory processes and novel, targeted therapies should be investigated. We suggest monitoring lipid levels in patients with PD to explore a potential pathogenic link. The proposed combined therapy, including IVIG, LMWH, vitamin C, topical proline, and topical tacrolimus is promising, however, it requires long-term follow-up.

\section{Abbreviations \\ ANA: Antinuclear antibody; anti-TPO: Anti-thyroid peroxidase; CBCd: Complete blood count and differential; CRP: C-reactive protein; ESR: Erythrocyte sedimentation rate; GH: Growth hormone; HSCT: Hematopoietic stem cell transplantation; IVIG: Intravenous immunoglobulin; LMWH: LoW molecular weight heparin; MBL: Mannose-binding lectin; NK: Natural killer; pBM: Basement membrane of the pial meninges; PBMC: Peripheral blood mononuclear cell; PD: Prolidase deficiency; Pt1: Patient 1; Pt2: Patient $2 ; T_{C M}$ : T central memory; $T_{\text {eff }}: T$ effector; $T_{E M}: T$ effector memory; $T_{E M R A}: T$ effector memory re-expressing CD45RA; $T_{\text {fh }}: T$ follicular helper; Treg: T regulatory; $S A A$ : Serum amyloid A; WISC-V: Wechsler Intelligence Scale for Children: 5th Edition.}

\section{Acknowledgements}

We would like to thank Dr. Erica Schollenberg, Department of Pathology \& Laboratory Medicine IWK Health Centre. We also thank the patients and their family.

\section{Authors' contributions}

$\mathrm{BD}$ and NA designed the study and wrote the first draft of the manuscript. $\mathrm{BD}, \mathrm{NA}$ and $\mathrm{AH}$ participated to the diagnostic procedures and clinical care of the patients. TI, BD, NA, SM, SR and DS were involved in the immunological analyses. TI and DS critically reviewed the manuscript. ZL performed the lonExchange Chromatography. All authors contributed to the preparation of the manuscript. All authors read and approved the final manuscript. 


\section{Funding}

Not applicable.

\section{Availability of data and materials}

Data are available upon request from the corresponding author.

\section{Declarations}

\section{Ethics approval and consent to participate}

The study was approved by the Nova Scotia Health Authority Research Ethics Board. All participants provided written informed consent.

\section{Consent for publication}

The patients gave consent to publication.

\section{Competing interests}

The authors declare that they have no competing interests.

\section{Author details}

${ }^{1}$ Division of Immunology, Department of Paediatrics, Dalhousie University, IWK Health Centre, Halifax, Canada. ${ }^{2}$ Division of Rheumatology, Department of Paediatrics, Dalhousie University, IWK Health Centre, Halifax, Canada. ${ }^{3}$ Division of Clinical Biochemistry \& Maritime Newborn Screening, Department of Pathology and Laboratory Medicine, Dalhousie University, IWK Health Centre, Halifax, Canada.

Received: 27 October 2021 Accepted: 9 February 2022

Published online: 23 February 2022

\section{References:}

1. Goodman SI, Solomons CC, Muschenheim F, McIntyre CA, Miles B, O'Brien D. A syndrome resembling lathyrism associated with iminodipeptiduria. Am J Med. 1968:45(1):152-9.

2. Spodenkiewicz M, Spodenkiewicz M, Cleary M, Massier M, Fitsialos G, Cottin V, et al. Clinical genetics of prolidase deficiency: an updated review. Biology (Basel). 2020;9(5):1-18.

3. Ferreira CR, Wang H. Prolidase deficiency. In: Adam MP, Ardinger $H_{H}$ Pagon RA, Wallace SE, Bean LJH, Gripp KW, Mirzaa GM, Amemiya A, editors. GeneReviews ${ }^{\circledR}$ [Internet]. Seattle (WA): University of Washington, Seattle; pp. 1993-2022.

4. Hershkovitz T, Hassoun G, Indelman M, Shlush LI, Bergman R, Pollack S, et al. A homozygous missense mutation in PEPD encoding peptidase D causes prolidase deficiency associated with hyper-lgE syndrome. Clin Exp Dermatol. 2006;31(3):435-40.

5. Falik-Zaccai TC, Khayat M, Luder A, Frenkel P, Magen D, Brik R, et al. A broad spectrum of developmental delay in a large cohort of prolidase deficiency patients demonstrates marked interfamilial and intrafamilial phenotypic variability. Am J Med Genet. 2010;153(1):46-56.

6. Rayment JH, Jobling R, Bowdin S, Cutz E, Dell SD. Prolidase deficiency diagnosed by whole exome sequencing in a child with pulmonary capillaritis. ERJ Open Res. 2019;5(2):00205-2018.

7. Bousfiha A, Jeddane L, Picard C, Al-Herz W, Ailal F, Chatila T, et al. human inborn errors of immunity: 2019 update of the IUIS phenotypical classification. J Clin Immunol. 2020;40(1):66-81.

8. Butbul Aviel Y, Mandel H, Avitan Hersh E, Bergman R, Adiv OE, Luder A, et al. Prolidase deficiency associated with systemic lupus erythematosus (SLE): single site experience and literature review. Pediatr Rheumatol. 2012;10(1):18.

9. Chasset F, Richez C, Martin T, Belot A, Korganow AS, Arnaud L. Rare diseases that mimic systemic lupus erythematosus (Lupus mimickers). Jt Bone Spine. 2019;86(2):165-71.

10. Sato S, Ohnishi T, Uejima Y, Furuichi M, Fujinaga S, Imai K, et al. Induction therapy with rituximab for lupus nephritis due to prolidase deficiency. Rheumatology. 2020;59(10): e57-9.

11. Kurien BT, D'Sousa A, Bruner BF, Gross T, James JA, Targoff IN, et al. Prolidase deficiency breaks tolerance to lupus-associated antigens. Int J Rheum Dis. 2013;16(6):674-80.
12. Costa-Reis P, Sullivan KE. Monogenic lupus: it's all new! Curr Opin Immunol. 2017:49:87-95.

13. Dunn R, Varigos $G$, Winship I. A photographic essay of prolidase deficiency. Clin Dysmorphol. 2011;20(4):194-9.

14. Kurien BT, D'Sousa A, Bruner BF, Gross T, James JA, Targoff IN, et al. Prolidase deficiency breaks tolerance to lupus-associated antigens. Int J Rheum Dis. 2013;16(6):678.

15. Lupi A, Rossi A, Campari E, Pecora F, Lund AM, Elcioglu NH, et al. Molecular characterisation of six patients with prolidase deficiency: identification of the first small duplication in the prolidase gene and of a mutation generating symptomatic and asymptomatic outcomes within the same family. J Med Genet. 2006;43(12):1-7.

16. Hui K-S, Lajtha A. Activation and inhibition of cerebral prolidase. J Neurochem. 1980;35(2):489-94.

17. Solak B, Kara RO, Erdem T, Muftuoglu T. A case of prolidase deficiency accompanying leg ulcers. Int J Low Extrem Wounds. 2015;14(1):92-4.

18. Freij BJ, Der Kaloustian VM. Prolidase deficiency: a metabolic disorder presenting with dermatologic signs. Int J Dermatol. 1986;25(7):431-3.

19. Wilk P, Uehlein M, Piwowarczyk R, Dobbek H, Mueller U, Weiss MS. Structural basis for prolidase deficiency disease mechanisms. FEBS J. 2018;285(18):3422-41.

20. Besio R, Maruelli S, Gioia R, Villa I, Grabowski P, Gallagher O, et al. Lack of prolidase causes a bone phenotype both in human and in mouse. Bone. 2015;72:53-64. https://doi.org/10.1016/j.bone.2014.11.009.

21. Kikuchi S, Tanoue A, Endo F, Wakasugi S, Matsuo N, Tsujimoto G. A novel nonsense mutation of the PEPD gene in a Japanese patient with prolidase deficiency. J Hum Genet. 2000;45(2):102-4.

22. Ferreira CR, Cusmano-Ozog K. Spurious elevation of multiple urine amino acids by ion-exchange chromatography in patients with prolidase deficiency. In: Morava E, Baumgartner M, Patterson M, Rahman S, Zschocke J, Peters V, editors. JIMD Reports, Volume 31. Berlin, Heidelberg: Springer; 2017. p. 45-9.

23. Pandit RA, Chen CJ, Butt TA, Islam N. Identification and analysis of a novel mutation in PEPD gene in two Kashmiri siblings with prolidase enzyme deficiency. Gene. 2013;516(2):316-9. https://doi.org/10.1016/j.gene.2012. 12.070 .

24. Slauenwhite D, MCAlpine SM, Hanly JG, Malik A, Haidl ID, Marshall JS, et al. Association of a Type 2-Polarized T Cell Phenotype with methotrexate nonresponse in patients with rheumatoid arthritis. Arthritis Rheumatol. 2020;72(7):1091-102

25. Shrinath M, Walter JH, Haeney M, Couriel JM, Lewis MA, Herrick AL. Prolidase deficiency and systemic lupus erythematosus. Arch Dis Child. 1997;76(5):441-4.

26. Cleary MA, Heaney M, Couriel JM, Walter JH. Immune function in prolidase deficiency. J Inherit Metab Dis. 1994;17(3):345-8.

27. Lubick KJ, Robertson SJ, McNally KL, Freedman BA, Rasmussen AL, Taylor $R T$, et al. Flavivirus antagonism of type i interferon signaling reveals prolidase as a regulator of IFNAR1 surface expression. Cell Host Microbe. 2015;18(1):61-74. https://doi.org/10.1016/j.chom.2015.06.007.

28. Ma CS, Deenick EK, Batten M, Tangye SG. The origins, function, and regulation of T follicular helper cells. J Exp Med. 2012;209(7):1241-53.

29. Lopes I, Marques L, Neves E, Silva A, Taveira M, Pena R, et al. Prolidase deficiency with hyperimmunoglobulin E: a case report. Pediatr Allergy Immunol. 2002;13(2):140-2.

30. Cleary MA, Heaney M, Couriel JM, Walter JH, Alehashemi S, GoldbachMansky R, et al. A homozygous missense mutation in PEPD encoding peptidase D causes prolidase deficiency associated with hyper-lgE syndrome. Clin Exp Dermatol. 2012;72(3):1091-102.

31. Ma CS, Deenick EK. The circulating life of a memory T-follicular helper cell. Clin Transl Immunol. 2017;6(5):e141.

32. Ahsan Rizvi S, Elder M, Beasley G. A novel manifestation of prolidase deficiency in a toddler diagnosed with very-early-onset crohn disease. J Pediatr Gastroenterol Nutr. 2019;69(3):e89-90.

33. Klar A, Navon-Elkan P, Rubinow A, Branski D, Hurvitz H, Christensen E, et al. Prolidase deficiency: It looks like systemic lupus erythematosus but it is not. Eur J Pediatr. 2010;169(6):727-32.

34. Mohr A, Atif M, Balderas R, Gorochov G, Miyara M. The role of FOXP3+ regulatory $T$ cells in human autoimmune and inflammatory diseases. Clin Exp Immunol. 2019;197(1):24-35. 
35. McKinney EF, Lee JC, Jayne DRW, Lyons PA, Smith KGC. T-cell exhaustion, co-stimulation and clinical outcome in autoimmunity and infection. Nature. 2015;523(7562):612-6.

36. Källström E, Roscher I, Andreasson A, Bäck O, Källström E, Roscher I, et al. Decreased frequency of intracellular IFN-g producing $T$ cells in whole blood preparations from patients with atopic dermatitis. Exp Dermatol. 2002;11(6):556-63.

37. Alehashemi S, Goldbach-Mansky R. Human autoinflammatory diseases mediated by NLRP3-, Pyrin-, NLRP1-, and NLRC4-inflammasome dysregulation updates on diagnosis, treatment, and the respective roles of IL-1 and IL-18. Front Immunol. 2020;11:1840.

38. Misiura M, Miltyk W. Current understanding of the emerging role of prolidase in cellular metabolism. Int J Mol Sci. 2020;21(16):1-20.

39. Surazynski A, Miltyk W, Palka J, Phang JM. Prolidase-dependent regulation of collagen biosynthesis. Amino Acids. 2008;35(4):731-8.

40. Mitchell S, Vargas J, Hoffmann A. Signaling via the NFkB system. Wiley Interdiscip Rev Syst Biol Med. 2016;8(3):227-41.

41. Willer CJ, Schmidt EM, Sengupta S, Peloso GM, Gustafsson S, Kanoni S, et al. Discovery and refinement of loci associated with lipid levels. Nat Genet. 2013:45(11):1274-85.

42. Insolia V, Priori EC, Gasperini C, Coppa F, Cocchia M, lervasi E, et al. Prolidase enzyme is required for extracellular matrix integrity and impacts on postnatal cerebellar cortex development. J Comp Neurol. 2020:528(1):61-80.

43. Royce PM, Danks DM. Normal hydroxylation of proline in collagen synthesized by skin fibroblasts from a patient with prolidase deficiency. J Inherit Metab Dis. 1982;5(2):111-3.

44. Isazade A, Elçin G, Doğan S, Gülseren D, Gököz Ö, Gürbüz B, et al. A rare cause of cutaneous ulceration: Prolidase deficiency. Int Wound J. 2019;16(4):1057-8.

45. Dunn R, Dolianitis C. Prolidase deficiency: the use of topical proline for treatment of leg ulcers. Australas J Dermatol. 2008;49(4):237-8.

46. Monafo V, Marseglia GL, Maghnie M, Dyne KM, Cetta G. Transient beneficial effect of $\mathrm{GH}$ replacement therapy and topical $\mathrm{GH}$ application on skin ulcers in a boy with prolidase deficiency. Pediatr Dermatol. 2000;17(3):227-30.

47. Karthikeyan K, Polly D, Asmathulla S, Balamurugan R, Kaviraj M. Topical proline therapy in prolidase deficiency. Clin Exp Dermatol. 2019;44(3):344-6.

48. Good AJ, Nielson CB, Schoch JJ. Topical tacrolimus therapy in the management of lower extremity ulcers due to prolidase deficiency. Pediatr Dermatol. 2019;36(6):926-8.

49. Süßmuth K, Metze D, Muresan AM, Lehmberg K, Zur Stadt U, Speckmann $C$, et al. Ulceration in prolidase deficiency: successful treatment with anticoagulants. Acta Derm Venereol. 2020;100(1):10-1.

50. Olasz L, Nyárády Z. The role of platysma-based flaps in the reconstruction of massive through and through facial defects. J Plast Reconstr Aesthetic Surg. 2008;61(10):1254-6.

51. Beradesca E, Fideli D, Bellosta M, Dyne KM, Zanaboni G, Cetta G. Blood transfusions in the therapy of a case of prolidase deficiency. Br J Dermatol. 1992;126(2):193-5.

52. Bakhtiar S, Fekadu J, Seidel MG, Gambinieri E. Allogeneic hematopoietic stem cell transplantation for congenital immune dysregulatory disorders. Front Pediatr. 2019;7:461.

\section{Publisher's Note}

Springer Nature remains neutral with regard to jurisdictional claims in published maps and institutional affiliations.

Ready to submit your research? Choose BMC and benefit from:

- fast, convenient online submission

- thorough peer review by experienced researchers in your field

- rapid publication on acceptance

- support for research data, including large and complex data types

- gold Open Access which fosters wider collaboration and increased citations

- maximum visibility for your research: over $100 \mathrm{M}$ website views per year

At BMC, research is always in progress.

Learn more biomedcentral.com/submissions 